Disponível em

http://www.anpad.org.br/rac

RAC, Rio de Janeiro, v. 18, n. 2, art. 1,

pp. 118-138, Mar./Abr. 2014

$(c)$ EY-No

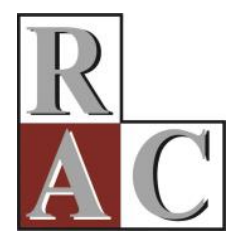

\title{
Percepção dos Funcionários sobre Mudanças Transacionais e Transformacionais em uma Organização Pública
}

\section{Employee Perception on Transactional and Transformational Changes in a Public Organization}

\author{
Sérgio Gabriel Domingos \\ E-mail: sgdomingos@gmail.com \\ Faculdades ICESP Promove de Brasília \\ QE 11 Área Especial, Guará, 71020-621, Brasília, DF, Brasil. \\ Elaine Rabelo Neiva \\ E-mail: elaine_neiva@uol.com.br \\ Universidade de Brasília - PSTO/UnB
} ICC Sul, Instituto de Psicologia, Sala AT-013, 70900-910, Brasília, DF, Brasil.

Artigo recebido em 02.10.2012. Última versão recebida em 04.09.2013. Aprovado em 13.10.2013. 


\title{
Resumo
}

Mudanças Transacionais alteram apenas alguns aspectos da organização. As Transformacionais são mais radicais, envolvendo a organização como um todo. A existência na organização de fatores de capacidade organizacional para mudanças pode exercer influência na ocorrência desses tipos de mudanças. Este trabalho avalia se a existência desses fatores influencia a percepção dos indivíduos sobre as mudanças transacionais e as transformacionais ocorridas num órgão público. A organização pesquisada é responsável pelas infraestruturas rodoviária, ferroviária e aquaviária, se passou por reestruturação, fusão e outras mudanças. Os dados $(\mathrm{N}=340)$ foram coletados na sede em Brasília, onde trabalham cerca de 450 indivíduos. Foram aplicados o Instrumento de Capacidade Organizacional para Mudanças - visando identificar a existência de fatores que facilitam a ocorrência de mudanças nas organizações -, e o Instrumento de Percepção de Mudanças Organizacionais, como medida para as mudanças organizacionais. Os resultados indicam que a estratégia de atuação, a dinâmica de coalizões e o fluxo de comunicação $\left(\mathrm{R}^{2}=0,35 ; \mathrm{p}=0,011\right)$ são preditores de percepção de mudanças Transformacionais. O trabalho em grupo $\left(\mathrm{R}^{2}=0,16 ; \mathrm{p}=0,001\right)$ aparece fracamente como preditor de mudanças do tipo Transacionais. A relevância da pesquisa está direcionada para aqueles que são responsáveis pela gestão administrativa de instituições públicas em processo de mudança.

Palavras-chaves: mudanças organizacionais; capacidade organizacional para mudanças; percepção de mudanças; tipologia de mudanças.

\begin{abstract}
Transactional changes alter only certain aspects of the organization. Transformational changes are more radical, involving the organization as a whole. The existence of capacity factors for organizational change in an organization can influence the occurrence of these types of changes. Change occurrences can be hindered or enhanced due to some organizational capacity factors, including information flow, organizational strategy, coalition dynamics, turbulence in the external environment, group autonomy, bureaucracy and managerial practices. The present paper aims to appraise whether these factors influence or not the way individuals perceive transformational and transactional changes in an organization. The data $(\mathrm{N}=340)$ have been gathered from a number of questionnaires applied in a public organization that has undergone management changes and had some of its areas restructured. The Organizational Capacity Assessment Scales for Changes and the Change Perception Scale have also been used. The results point out that organizational strategy, coalition dynamics and communication flow $(\mathrm{R} 2=0.35 ; \mathrm{p}=0.011)$ are predictors for Transformational Changes perception. As for Transactional Changes, group work $(\mathrm{R}=0.16 ; \mathrm{p}=0.001)$ is barely considered a predictive factor. The relevance of this research is directed toward those who are responsible for the management process in public institutions.
\end{abstract}

Key words: organizational change capacity; change perception; change evaluation; organizational change; change typology. 


\section{Introdução}

Eisenhardt e Martin (2000) salientam que as organizações que tiveram condições de se manter vivas foram aquelas que tiveram habilidade em responder rápida e efetivamente às condições impostas pela mudança.

De acordo com Burke (2011a, 2011b) e Burke e Litwin (1992), na busca de respostas eficientes, as organizações efetuam mudanças utilizando dois caminhos: de forma paulatina - alterando apenas alguns aspectos de sua estrutura, o que caracteriza uma mudança do tipo Transacional; ou efetuando uma mudança radical, com a reconfiguração de toda a organização, a ruptura dos padrões existentes, a criação de novas estratégias e de uma nova missão - o que caracteriza uma mudança do tipo Transformacional. Tais ocorrências têm a influência positiva ou negativa de fatores de capacidade organizacional para mudanças.

A capacidade organizacional para a mudança está vinculada à existência de condições ou características organizacionais, ou dos seus membros, que são positivamente relacionados à implantação de mudanças ou que funcionem como facilitadores do estabelecimento dessa mudança (Gravenhorst, Werkman, \& Boonstra, 2003).

Num processo de mudança organizacional, vários fatores podem facilitar ou dificultar a ocorrência de mudanças (Pettigrew, Woodman, \& Cameron, 2001).

Os trabalhos de Neiva e Domingos (2011) e Fischer e Lima (2005) apresentam fatores de capacidade organizacional para mudança que podem exercer influência no processo: o fluxo de informação, a existência de uma estratégia de atuação organizacional, as coalizões que apoiem a mudança, a turbulência no ambiente externo, a autonomia de grupos, a burocracia, o trabalho em grupo e a confiança na atuação gerencial.

Do ponto de vista teórico, fatores que auxiliam ou dificultam o processo de mudanças estariam associados a uma percepção mais efetiva de mudanças na organização, sendo a avaliação perceptual uma das formas de se mensurar essas alterações. Salienta-se que a maioria das formas de mensuração desse fenômeno se dá a partir dos resultados numéricos (dados brutos) das intervenções, o que muitas vezes não gera mudanças percebidas pelos indivíduos (Glor, 2007).

A identificação de fatores que possam influenciar a mudança e o conhecimento da percepção dos seus membros sobre a existência ou não desses fatores na organização são pontos relevantes para todos aqueles responsáveis pela gestão administrativa de instituições públicas em processo de mudança, especialmente no que se refere ao gerenciamento das pessoas antes, durante e depois da implantação desse processo.

Os autores argumentam que as contribuições teóricas e práticas apresentadas neste trabalho possam auxiliar os gestores na análise e na condução de processos de mudanças, em especial, nas instituições públicas.

Dessa forma, este trabalho objetiva avaliar se os fatores de capacidade organizacional para mudanças transformacionais e os fatores de capacidade organizacional para mudanças transacionais influenciam a percepção da ocorrência desses tipos de mudança num órgão público.

O órgão público pesquisado atualmente é responsável por construção, manutenção e operação de infraestrutura dos modos rodoviário, ferroviário e aquaviário. Possui aproximadamente, 1200 funcionários. Tem sua sede em Brasília/DF (local deste estudo), onde trabalham cerca de 450 indivíduos. A organização passou por mudanças profundas - absorção da unificação de vários órgãos públicos - que transformaram de forma radical a sua natureza, alteraram a sua missão, reconfiguraram as atividades desenvolvidas e os processos de trabalho. Antes da unificação, a organização era responsável apenas pela manutenção de rodovias, possuía um contingente com conhecimento 
específico e sua estrutura organizacional estava direcionada para o ideal atendimento dessa área. Com a absorção dos órgãos responsáveis pelas áreas aquaviária e ferroviária, a organização foi descaracterizada da sua missão inicial. A sua estrutura foi reorganizada para adaptar-se às novas atividades. Essa restruturação envolveu todo o órgão: os setores de trabalho, as estratégias de atuação e, principalmente, o modelo de gestão de pessoas, que, com a unificação, passou a contar, no mesmo espaço físico, com indivíduos possuidores de conhecimentos específicos sobre áreas diversas. Durante o processo de unificação, além dessas mudanças de grande impacto, a organização também sofreu pequenas mudanças internas que influenciaram nas relações de trabalho. O conhecimento de todo esse processo de mudança foi possível graças às informações colhidas por meio de entrevistas realizadas com os membros que vivenciaram esses processos.

\section{Conceitos de mudança organizacional e tipos de mudanças}

A conceituação de mudança organizacional tem sido proposta por diversos autores, como Woodman (1989); Barnett e Carroll (1995); Van de Ven e Poole (2005), que entendem o processo de mudança como ocorrências comparadas em diferentes períodos de tempo. Ou seja, envolvem a comparação antes e depois da transformação.

Neiva e Paz (2007, 2012), ampliando a conceituação proposta por Lima e Bressan (2003), entendem mudança organizacional como

qualquer alteração, planejada ou não, em componentes que caracterizam a organização como um todo - finalidade básica, pessoas, trabalho, estrutura formal, cultura, relação da organização com o ambiente - decorrente de fatores internos e/ou externos à organização, que traz alguma consequência, positiva ou negativa, para os resultados organizacionais ou para sua sobrevivência. (Neiva \& Paz, 2007, p. 32)

Segundo Choi e Ruona (2011), a mudança organizacional é definida conforme a perspectiva utilizada pelo pesquisador. Sob a perspectiva da gestão estratégica, os autores a apresentam como um processo de implementação da estratégia corporativa desenvolvida pelos líderes organizacionais e tomadores de decisão. Sob a perspectiva do desenvolvimento organizacional, ela é percebida como um conjunto de esforços intencionais cuja finalidade é promover o desenvolvimento individual, melhorar o desempenho da organização e fazer a diferença no ambiente organizacional.

Várias conceituações podem ser adotadas quanto ao construto de mudança organizacional, contudo, todas elas se reportam a alterações realizadas e/ou percebidas na organização ao longo do tempo (Lima \& Bressan, 2003). Segundo Woodman (1989), toda medida é uma abstração. A mudança, na sua forma mais simplificada, é representada pela diferença ou pela comparação entre medidas tiradas em dois pontos do tempo. Em termos do adicionamento de significado aos fenômenos, o conceito de mudança se reporta à abstração de segunda ordem - tanto da perspectiva da psicometria quanto da perspectiva da mente que adiciona significado às coisas percebidas - que justifica o uso da percepção como sua medida.

Além da questão conceitual e da evolução do campo, outro ponto a ser considerado diz respeito à necessidade de se especificar qual o tipo de mudança está em discussão, bem como quais são o seu objeto e o seu conteúdo.

\section{Tipologias, objetos e conteúdos de mudança organizacional}

Armenakis e Bedeian (1999), em revisão da literatura sobre mudanças organizacionais, identificam que os diversos estudos nessa área procuram avaliar questões vinculadas aos conteúdos (o que muda na organização); aos contextos (das forças atuantes dentro e fora da organização); aos processos (ações que ocorrem durante o processo de mudança); e aos critérios (resultados comportamentais e afetivos da implantação da mudança). 
De acordo com os trabalhos de Lima e Bressan (2003) e Neiva e Paz (2007, 2012), algumas dimensões podem ser consideradas na apresentação de tipologias pertinentes a mudanças, como a continuidade ou a descontinuidade de tempo; o objeto, a intensidade, a velocidade, e a intencionalidade da mudança; o tempo de resposta ao meio externo, o papel dos envolvidos com o processo, como também o prazo no qual ocorre a mudança.

Weick e Quinn (1999) constatam que existem dois grandes tipos de mudanças que são analisados pela literatura: as mudanças Contínuas, que envolvem pequenos avanços ao longo do tempo, sendo cumulativas, e as mudanças Episódicas, resultado do desequilíbrio organizacional. Segundo Van de Ven e Poole (2005), essa diferenciação reflete tendências do estudo e visões de organizações, bem como abordagens metodológicas diferenciadas no estudo da mudança organizacional.

Burke (2011a, 2011b) e Burke e Litwin (1992) propõem uma diferenciação entre mudança Transformacional e mudança Transacional. Segundo os autores, a mudança Transformacional possui grande impacto, consequência da interação e das pressões do ambiente externo, demandando novos comportamentos dos empregados e alterando toda a organização. Envolve a ruptura de padrões anteriores, atingindo a organização em sua totalidade. Exige uma reconfiguração e um redirecionamento: a criação de novas estratégias, atividades, missão; a organização dos processos, de estrutura e de pessoas. Segundo os autores, o outro tipo de mudança - a mudança Transacional - é gerado pela atuação interna da organização sobre fatores individuais, grupais e organizacionais que estão relacionados às operações (transações) do dia a dia dos indivíduos no ambiente organizacional. Ela altera apenas alguns aspectos da organização e, pressupondo a estabilidade externa, faz pequenos ajustes continuamente visando à eficiência organizacional. Ela não exige alteração da identidade, dos valores ou da missão. Os tipos de mudanças Transformacionais e Transacionais apresentadas por Burke e Litwin (1992) correspondem, respectivamente, aos tipos de mudança Episódica e Contínua relatados por Weick e Quinn (1999).

A tipologia de Mudanças Transformacionais e Transacionais proposta por Burke e Litwin (1992) foi utilizada neste trabalho como balizamento na análise das mudanças ocorridas na organização pesquisada, pois estabelece conteúdos que possam ser usados para separar os tipos de mudanças.

Armenakis e Bedeian (1999) ressaltam que o contexto interno apresenta fatores que facilitam ou dificultam o processo de mudanças. A utilização neste trabalho de fatores que facilitam ou dificultam mudanças é necessária para o entendimento do que é identificado pelos membros da organização e da sua relação com a percepção da ocorrência de mudanças Transacionais e Transformacionais. Pettigrew, Woodman e Cameron (2001) afirmam que há poucos estudos empíricos que verificam a relação entre a capacidade de mudança e o desempenho organizacional, recomendando pesquisas nessa questão.

Segundo Judge e Douglas (2009) e Glor (2007), aproximadamente 70\% das iniciativas de mudanças organizacionais planejadas fracassam. Os primeiros autores apontam como um dos principais motivos desses fracassos a ausência de instrumentos de diagnóstico válidos e confiáveis para avaliar e acompanhar a capacidade organizacional para mudança.

\section{Capacidade organizacional para mudança}

O termo capacidade é definido nos dicionários e utilizado corriqueiramente vinculado às ideias de condição, de qualidade ou de potencial para se exercer uma atividade ou mesmo para se efetuar algum tipo de ação. Objetos e pessoas são frequentemente qualificados em função dos seus potenciais para a realização de algo a que se propõem de forma efetiva. No ambiente organizacional, o termo assume conotações semelhantes, vinculando-se às condições necessárias para a empresa ou para seus empregados atuarem de forma a adaptar-se e garantir suas sobrevivências. 
Greenwood e Hinings (1996) desenvolveram o conceito de capacidade para ação a fim de explicar as várias formas que as organizações têm de lidar com as pressões para mudança. Tal conceito envolve a existência de algumas características na organização ou de seus membros que permitem o desenvolvimento de uma ação no sentido de se efetuar mudança organizacional.

A partir daí, Slack e Hinings (2004) investigaram o papel dos interesses das unidades, das relações de poder e da capacidade organizacional em um programa de mudança transformacional em organizações esportivas canadenses. Os autores estudaram a dinâmica organizacional e seu papel propulsor e facilitador da mudança. Os autores ressaltam o apoio da liderança de topo e o empoderamento dos membros como um dos aspectos da capacidade organizacional para mudanças.

Litaker, Ruhe e Flocke (2008) realizaram um estudo em organizações hospitalares e identificaram que a motivação de empregados, recursos e suporte ao trabalho, ação da liderança, influências externas e uso das oportunidades de mudanças compõem a capacidade organizacional para mudanças. Os trabalhos de Slack e Hinings (2004), Litaker et al. (2008) focaram principalmente o indivíduo no ambiente organizacional.

Judge e Douglas (2009) identificaram alguns conceitos e medidas relacionados ao construto capacidade organizacional para mudança, mas consideram que "nenhum deles apreende totalmente a essência da capacidade organizacional para mudança de forma confiável e válida" (p. 636). Os autores examinaram os conceitos de flexibilidade organizacional, receptividade organizacional para mudanças, prontidão dos empregados para mudanças, adaptabilidade organizacional e aprendizagem organizacional como construtos similares a capacidade organizacional para mudanças.

A definição constitutiva de flexibilidade organizacional apontada por Hatum e Pettigrew (2004) pode ser considerada como a mais próxima à definição de capacidade organizacional para mudança de Judge e Douglas (2009). Ambas identificaram uma combinação de capacidades da organização e de gestão que possibilitam a adaptação mais rápida a uma mudança. No entanto, a operacionalização da definição de Hatum e Pettigrew (2004) envolvendo dimensões relacionadas à internacionalização e à diversificação de produtos torna-se bastante distinta das dimensões apresentadas por Judge e Douglas (2009).

Neiva e Paz (2012) ressaltam que existe uma confusão na literatura acerca dos conceitos de prontidão e capacidade organizacional para mudança. Autores como Prochaska, Norcross e DiClemente (1994), e Cunningham et al. (2002) entendem o construto capacidade para mudança como uma disposição para tal - readiness for organizational change - correlacionando-o ao sentido de prontidão, direcionando o olhar exclusivamente para as condições existentes nos indivíduos, as quais permitiriam ou não facilitar o processo de mudança em ambientes de atendimento à saúde. Assim, o conceito de prontidão está vinculado à percepção do indivíduo sobre as suas condições pessoais para a efetivação de um processo de mudança.

Weiner, Amick e Lee (2008) consideram prontidão organizacional para a mudança como um construto bidimensional que se refere à medida que os membros da organização estão psicológica e comportamentalmente preparados para implementar uma mudança organizacional. Em outras palavras, refere-se à motivação e à capacidade dos membros organizacionais de implementar mudança organizacional intencional. Tal posicionamento se compara aos apresentados por Slack e Hinings (2004), Litaker et al. (2008).

Oxtoby, McGuiness e Morgan (2002) analisaram onze organizações do Reino Unido e identificaram algumas características para o desenvolvimento da capacidade organizacional para mudanças. Os autores elencaram o estabelecimento de metas e objetivos, a mensuração de processos e a criação de equipes de responsáveis pelo desenvolvimento do projeto de mudanças como características fundamentais.

Para Buono e Kerber (2010), o conceito de capacidade organizacional para mudanças está associado ao desenvolvimento de um contexto de aprendizagem nas organizações. Esse contexto de 
aprendizagem não só encoraja os indivíduos a se engajar em aprendizagem organizacional, mas também aumenta a capacidade organizacional para fazer mudanças bem-sucedidas.

Judge e Elenkov (2005), em acordo com a teoria baseada na alocação de recursos (resourcebased view [RBV]), entendem capacidade para mudança como "uma ampla e dinâmica capacidade existente na organização, que permite a empresa adaptar as velhas capacidades às novas ameaças e oportunidades e assim criar novas capacidades" (p. 893). Segundo os autores, a organização e o meio interagem continuamente para estabelecer equilíbrio na dinâmica da situação. Como o objetivo é manter o equilíbrio, a organização constantemente se vê na necessidade de criar novas capacidades. Para Staber e Sydow (2002), essas capacidades foram chamadas anteriormente de capacidade adaptativa.

Nas visões de Judge e Elenkov (2005) e Judge e Douglas (2009), a capacidade de mudança organizacional possui oito dimensões distintas, mas inter-relacionadas, que são: a liderança e credibilidade, que envolvem a capacidade do líder de transmitir segurança e confiabilidade para os membros da organização; a confiança dos seguidores, que diz respeito à habilidade dos integrantes em discordar ou concordar construtivamente com os novos caminhos definidos pelos líderes; a habilidade da organização em atrair, reter e desenvolver lideranças; a habilidade da gerência em nível médio em estabelecer link entre a alta gerência e o restante dos membros da organização; uma cultura inovadora, em que a organização estabelece e encoraja novas ideias; uma cultura responsável, em que a organização administra responsavelmente os recursos e chega às metas determinadas; uma sistemática de comunicação vertical e horizontal com clientes e demais stakeholders; e uma sistemática de acompanhamento em que a organização foca nas raízes e causas do problema, e reconhece as interdependências dentro e fora dos limites da organização.

Autores como Judge e Elenkov (2005); Judge e Blocker (2008); Judge e Douglas (2009) têm se dedicado a sistematizar esse construto, construindo instrumentos para avaliá-lo e especificando fatores antecedentes e consequentes do fenômeno.

Para Gravenhorst, Werkman e Boonstra (2003, p. 86), capacidade de mudança é "o grau em que aspectos da organização e aspectos do processo contribuem para as mudanças ou as restringem". Segundo os autores, os aspectos da organização tanto quanto dos processos podem contribuir ou restringir mudanças, pois são inter-relacionados; a alteração em um deles afeta diretamente a outro. Os aspectos da organização que contribuem ou restringem mudanças encontram-se relacionados e conceituados na Tabela 1.

Tabela 1

\section{Aspectos da Organização que Contribuem ou Restringem Mudanças}

\begin{tabular}{ll}
\hline $\begin{array}{l}\text { Metas e Estratégias da organização (goals and strategy } \\
\text { of the organization) }\end{array}$ & $\begin{array}{l}\text { O conhecimento das metas e das estratégias } \\
\text { organizacionais por todos os seus membros. }\end{array}$ \\
Estrutura de trabalho (structure) & $\begin{array}{l}\text { Características da estrutura de trabalho (orgânica x } \\
\text { mecanicista). }\end{array}$ \\
Cultura organizacional (culture) & $\begin{array}{l}\text { Cultura organizacional direcionada à inovação e à } \\
\text { orientação. }\end{array}$ \\
Tecnologia na comunicação (technology) & $\begin{array}{l}\text { Presença de elevado nível de informação e uso da } \\
\text { tecnologia como ferramenta de comunicação. }\end{array}$ \\
Características do trabalho (job characteristcs) & $\begin{array}{l}\text { Grau de satisfação dos membros com o tipo de trabalho } \\
\text { realizado e as possibilidades de desenvolvimento. }\end{array}$ \\
Relações internas de poder (power relations) & $\begin{array}{l}\text { O balanço entre a autonomia e a dependência mútua de } \\
\text { grupos e indivíduos na organização. }\end{array}$
\end{tabular}

Nota. Fonte: Gravenhorst, K. M. B, Werkman, R. A., \& Boonstra, J. J. (2003). The change capacity of organisations: general assessment and five configurations (p. 93). Applied Psychology: An International Review, 52(1), 83-105. doi: 10.1111/14640597.00125 
De acordo com Gravenhorst et al. (2003), os aspectos do processo também podem exercer influência, contribuindo ou dificultando mudanças na organização. Na visão dos autores, esses aspectos geralmente não são levados em conta quando a capacidade de mudança organizacional é determinada. Começam a ser visualizados pelos membros somente após a organização já ter definido as metas de mudança e a forma de efetuá-las. Os aspectos do processo são apresentados e conceituados por meio da Tabela 2.

Tabela 2

\section{Aspectos do Processo que Contribuem ou Restringem Mudanças}

Metas e Estratégias da mudança (goals and strategy of change)

Aspectos tecnológicos (technological aspects)

Tensões e relações de poder dos grupos (tensions within and between groups in the organization)

O tempo do processo (the timing of the process)

Suporte de informações (information supply)

Geração de suporte para a mudança (generation of support for the change)

O papel dos gerentes da mudança (the role of change managers)

O papel dos gerentes de linha (the role of the line managers)

Resultados esperados (expected outcomes)

Suporte para mudança (support for change)
A transformação dos objetivos abstratos e das estratégias de mudança em ações concretas.

A integração tecnológica da empresa com o processo de mudança.

As tensões existentes geradas e entre grupos pró e contra a mudança.

Timing do processo de mudança, envolvendo a intensidade e o tempo de adaptação dos indivíduos com a mudança.

Fornecimento de informações claras e honestas a todos os membros da organização, com a utilização de várias mídias.

Geração de suporte e participação dos empregados.

O papel dos gestores de mudança envolvendo a credibilidade e a confiança, diminuindo a incerteza.

O papel dos gerentes de linha envolvendo a aplicação de ações concretas de mudança nos seus departamentos e equipes.

Os resultados (psicológicos) esperados. Condições que ajudam os indivíduos a desenvolver atitudes direcionadas à mudança.

O suporte dado pelos empregados ao processo de mudança; seu envolvimento por meio da utilização de ideias e participação na condução da mudança.

Nota. Fonte: Gravenhorst, K. M. B, Werkman, R. A., \& Boonstra, J. J. (2003). The change capacity of organisations: general assessment and five configurations (p. 93). Applied Psychology: An International Review, 52(1), 83-105. doi: 10.1111/14640597.00125

De acordo com Soparnot (2011, p. 640), “a capacidade para mudança de uma organização é a habilidade em produzir soluções (conteúdo) que respondam à evolução ambiental (contexto externo) e/ou à evolução organizacional (contexto interno) e em implementar esses processos de mudança com sucesso (processo)". Com base em estudo de caso na Renault S.A. e utilizando técnicas qualitativas (entrevistas, observações diretas e pesquisa documental), o autor propôs um modelo de capacidade para mudança. Ele identificou três dimensões da capacidade para mudança: contexto, processo e aprendizagem. A dimensão de contexto consiste nos recursos e nas habilidades que facilitam o processo de mudança (valor da mudança, flexibilidade estrutural, convergência cultural, confiança entre aqueles que sofrem a mudança e aqueles que a realizam, práticas baseadas no consenso e capacidade individual de aprendizagem). A dimensão de processo incorpora princípios de implementação da mudança (liderança transformacional, legitimidade percebida da mudança, construção participativa da mudança, desenvolvimento incremental e criação de visibilidade). A dimensão de aprendizagem lida com a "capacidade introspectiva" da organização (aperfeiçoamento 
por meio da experiência, renovação pela tentativa e compartilhamento de conhecimento entre departamentos).

Para Neiva e Paz (2007, 2012), Neiva e Domingos (2011), Gravenhorst et al. (2003), a capacidade organizacional para a mudança está vinculada à existência de condições ou características organizacionais, ou dos seus membros, que são positivamente relacionados à implantação de mudanças ou que funcionem como facilitadores do estabelecimento dessa mudança. Tal conceituação foi utilizada como base neste trabalho.

Segundo Neiva e Paz (2007, 2012), Neiva e Domingos (2011) e Fischer e Lima (2005), a capacidade organizacional para mudança envolve a presença de oito fatores preponderantes: o fluxo de informação abrangente, rápido e preciso dentro da organização; a existência de uma estratégia de atuação organizacional baseada na análise do ambiente externo; a existência de coalizões organizacionais apoiadoras da mudança; a existência de turbulência no ambiente externo; a presença de autonomia de unidades e pessoas na organização; a preponderância do trabalho em grupo, envolvendo pessoas e unidades; manifestação de direção, flexibilidade e confiança na atuação gerencial. A burocracia e o continuísmo no funcionamento organizacional foram considerados como fatores que restringem a introdução de mudanças na organização. Tais fatores foram levantados por meio de entrevistas e revisão de literatura conforme descritas no artigo de validação do instrumento Neiva e Paz (2007, 2012), Neiva e Domingos (2011), Fischer e Lima (2005).

Em relação aos modelos utilizados, os modelos de Soparnot (2011), de Judge e Douglas (2009) e o de Neiva e Domingos (2011) tratam de condições facilitadoras da mudança organizacional; os modelos de Gravenhorst et al. (2003) e o de Bouckenooghe, Devos e Van Den Broeck (2009) abordam a gestão da mudança.

Danna (2012), realizando um levantamento na literatura das características organizacionais que podem facilitar ou restringir mudanças, identificou 17 grupos de características: comunicação, ambiente externo, grupos que influenciam o processo de mudanças, hierarquia organizacional, liderança, trabalho em equipe, funcionamento organizacional, participação dos empregados, gestão da mudança, alinhamento de percepções sobre a capacidade organizacional para mudança, cultura organizacional, tecnologia, condições de trabalho, estrutura organizacional, estratégia organizacional, características e percepções dos funcionários e aprendizagem na organização.

Neiva e Domingos (2011), a partir da literatura existente e da análise de conteúdo das entrevistas com gestores, dividem os fatores de capacidade organizacional para mudanças em Transformacionais e Transacionais, de acordo com o impacto desses sobre o escopo e tempo/velocidade dos processos de mudanças organizacionais. Segundo os autores, quatro são os fatores que podem influenciar na implantação de mudanças Transformacionais. Esses fatores se encontram relacionados na Figura 1.

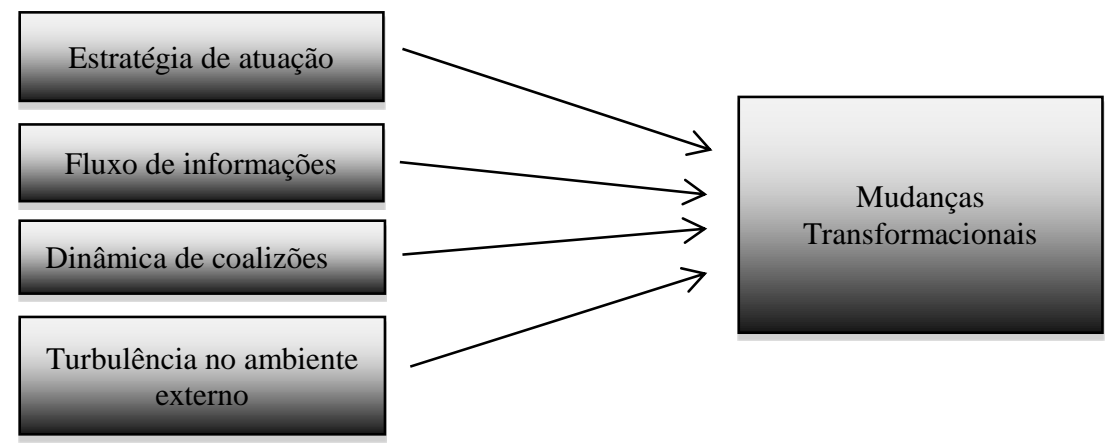

Figura 1. Características Associadas a Mudanças Transformacionais. 
A Figura 2 mostra os fatores que estariam associados a mudanças Transacionais.

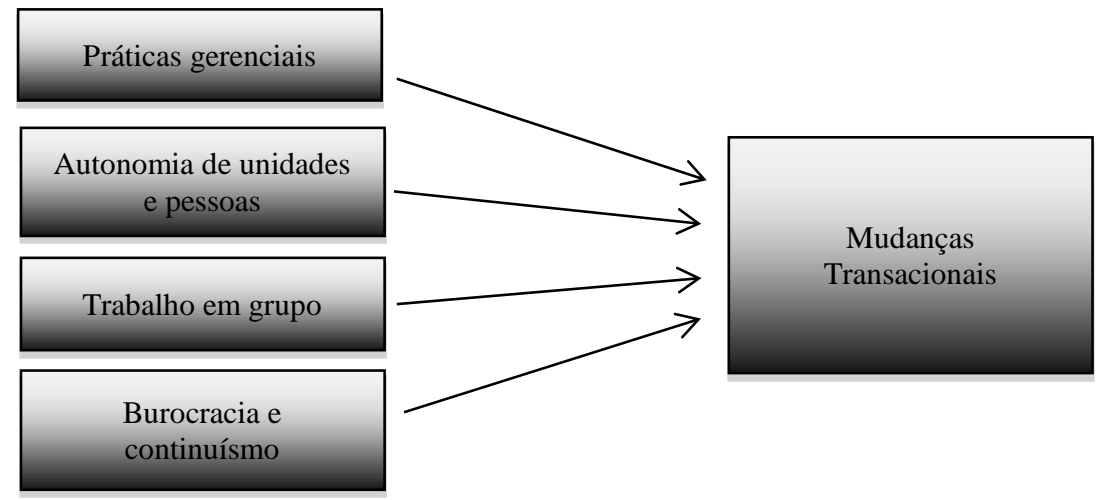

Figura 2. Características Associadas a Mudanças Transacionais.

Considerando a divisão das características de capacidade organizacional para mudanças em Transformacionais e Transacionais, a seguinte questão foi levantada: Os fatores de capacidade organizacional para mudanças transformacionais e transacionais influenciam a percepção da ocorrência desses tipos de mudança no ambiente organizacional? Dessa forma, a pesquisa objetivou verificar se as características organizacionais afetam a ocorrência de mudanças na organização avaliadas por meio da percepção dos seus membros.

\section{Método}

A presente pesquisa se caracteriza por ser um recorte transversal (Sampieri, Collado, \& Lúcio, 2006), cuja coleta de dados foi realizada por questionários numa abordagem metodológica do tipo Survey. De acordo com Sampieri, Collado e Lúcio (2006), trata-se de um estudo de natureza correlacional que pretende associar as variáveis componentes da capacidade organizacional para mudanças, às mudanças percebidas pelos membros da organização estudada.

A organização escolhida é um órgão executor da política de transportes do Governo Federal, implantada em 2002, para desempenhar as funções relativas a construção, manutenção e operação de infraestrutura dos modos rodoviário, ferroviário e aquaviário. Possui unidades em todo o país, totalizando, aproximadamente, 1200 funcionários. Tem sua sede em Brasília (DF), onde trabalham, entre funcionários e terceirizados, um contingente de 450 indivíduos. A escolha se deu pelo fato da organização ter passado por mudanças (conforme conceito apresentado) nos dois últimos anos.

A descrição das mudanças foi inicialmente identificada por alguns informantes-chave que vivenciaram esses processos. Segundo estes, as mudanças de grande porte (transformacionais) ocorridas na organização pesquisada dizem respeito ao processo de unificação de alguns órgãos que desempenhavam atividades relativas a construção, manutenção e operação de infraestrutura nas áreas terrestre (rodovias, ferrovias, viadutos etc.), marítima e lacustre (portos, canais etc.).

Antes da unificação, o órgão pesquisado tinha sob a sua responsabilidade apenas a administração das rodovias. Com a unificação, teve que absorver a administração de outros modais. Tais mudanças geraram na organização uma situação complexa: a junção de indivíduos com conhecimentos de áreas distintas e especializações completamente diferentes, e que, a partir daquele momento, encontravam-se na obrigação de desenvolver atividades diversas, muitas vezes diferentes das quais tinham pleno domínio. Em consequência do processo de unificação, pequenas mudanças (transacionais) ocorreram ao longo do tempo, como a troca de gestores e pontuais alterações no ambiente e na forma de trabalho. Tais mudanças, conforme relatos obtidos, também geraram 
insegurança e descontentamento, principalmente em função da escolha de gestores que não possuíam conhecimento técnico, mas eram politicamente articulados, e das constantes alterações de rumos e indefinições no estabelecimento de metas.

\section{Amostra}

O número de respondentes alcançados neste estudo foi de $\mathrm{N}=340$, o que corresponde a $75,6 \%$ do total, com exclusão de indivíduos em férias, afastamentos por motivos de saúde, viagens, congressos e outras atividades externas, o que sinaliza uma representatividade da amostra em relação à população (membros da organização). De acordo com o G. Power, para um tamanho de efeito estimado em 0,15, um Alfa de 0,05 e um poder de teste de 0,95, tendo 8 preditores na regressão múltipla e usando o Teste T Bi caudal, é necessária uma amostra mínima de 89 sujeitos para a realização da pesquisa. Portanto, requisitos de representatividade e poder a priori estimado do teste foram alcançados por esta pesquisa.

Quanto ao sexo, a distribuição indicou maioria masculina $(\mathrm{n}=196)$, correspondendo a $58 \%$ da amostra. Em relação à idade, os respondentes apresentaram idade média de 39,6 anos $(\mathrm{SD}=11,9)$, tendo o mais novo 22 anos e o mais idoso 79 anos. As faixas etárias com maior frequência apareceram com 28 anos $(n=17)$ e 30 anos $(n=16)$. O tempo médio de serviço foi de 10,1 anos $(\mathrm{SD}=9,65)$. Salienta-se, contudo, o elevado número de respondentes com poucos anos de trabalho; 2 anos $(\mathrm{n}=61)$ ou 3 anos $(\mathrm{n}=55)$. Quanto ao tipo de cargo ocupado, a maioria encontra-se vinculada à execução da área-meio $(\mathrm{n}=145)$.

\section{Instrumento}

Dois instrumentos foram aplicados. O Instrumento de Capacidade Organizacional para Mudança foi elaborado por Neiva e Paz (2007), baseando-se nos trabalhos de Fischer e Lima (2005), e validado em cinco organizações brasileiras com 1295 respondentes (Neiva \& Domingos, 2011). Visa identificar a existência na organização de fatores relacionados às mudanças transformacionais ou transacionais. O instrumento é constituído por duas escalas, totalizando 98 itens, sendo ambas compostas por fatores que abordam aspectos que facilitam ou dificultam o processo de mudança organizacional. A primeira escala aborda os fatores facilitadores que propulsionam as mudanças transformacionais, e, a segunda, apresenta os fatores propulsores de mudanças transacionais. As duas escalas possuem índices de validade satisfatórios, como cargas fatoriais acima de 0,50 e alfas de Cronbach dos fatores acima de 0,75 .

Paralelamente, foi aplicado o Instrumento de Percepção de Mudanças Organizacionais, visando identificar se o indivíduo percebe as mudanças transformacionais ou transacionais que ocorreram na organização. O instrumento é composto de um conjunto de 30 frases (itens) disposto aleatoriamente, numa escala de concordância, tipo Likert, de 10 pontos. Essa escala foi criada e validada para este trabalho.

\section{Procedimentos de coleta e análise de dados}

Os dados foram coletados por meio de um questionário aplicado coletivamente aos respondentes. As reuniões de aplicação duraram em média 30 minutos e foram conduzidas para grupos de 25 membros da organização pesquisada. Os dados foram processados por meio do programa estatístico Statistical Package for the Social Science (SPSS), versão 16.0.

Foram realizadas análises estatísticas descritivas e inferenciais. O comportamento das variáveis foi analisado por médias e desvios-padrão. Foram executadas regressões múltiplas padrão para avaliar a influência dos fatores de capacidade organizacional para mudanças sobre as alterações percebidas pelos membros da organização. 
Cinco regressões múltiplas foram efetuadas. A primeira teve por objetivo verificar se havia influência das variáveis demográficas sobre as variáveis-critério (percepção de mudanças transacionais e percepção de mudanças transformacionais). A segunda e a terceira regressões tiveram como variáveis-critério a percepção de mudanças transacionais e transformacionais, e como variáveis preditoras os respectivos fatores transacionais e transformacionais de capacidade organizacional. A quarta e a quinta regressões foram realizadas para verificar se todos os fatores de capacidade organizacional eram preditores das mudanças transformacionais e transacionais percebidas.

Apesar dos indícios de validade apresentados pelos autores, os instrumentos foram submetidos à análise fatorial para verificar sua validade e sua confiabilidade na amostra da pesquisa. Para extração dos fatores, foi utilizada a análise fatorial dos eixos principais (PAF - Principal Axis Factoring) - e a rotação pelo método Promax.

\section{Resultados}

Os resultados encontram-se apresentados na seguinte ordem: dados de validação das escalas, dados descritivos das variáveis da pesquisa e resultados do teste com as relações hipotetizadas pela pesquisa.

\section{Dados de validação dos instrumentos para a amostra da pesquisa}

\section{Instrumento de capacidade organizacional para mudanças}

As duas subescalas de capacidade organizacional para mudança foram submetidas novamente à análise fatorial (método dos eixos principais e rotação Promax) para verificar se a estrutura inicial se mantinha para a atual amostra. De acordo com os resultados, para a amostra desta pesquisa, a variância total explicada pelas duas subescalas foi de $58 \%$ e $52 \%$, respectivamente.

A primeira escala aborda os resultados dos fatores facilitadores que propulsionam as mudanças transformacionais - (1) fluxo de informação; (2) estratégias de atuação; (3) dinâmicas de coalizões; e (4) turbulência no ambiente externo, de acordo com Neiva e Domingos (2011). Essa escala encontrase apresentada na Tabela 3.

Tabela 3

Estrutura Fatorial dos Fatores Transformacionais que Facilitam ou Inibem a Mudança Organizacional

\begin{tabular}{|c|c|c|c|c|}
\hline \multirow{2}{*}{ Itens* } & \multicolumn{4}{|c|}{ Fatores } \\
\hline & 1 & 2 & 3 & 4 \\
\hline As informações atingem igualmente todos os servidores da empresa. & 0,861 & & & \\
\hline As informações são disseminadas para os servidores com rapidez. & 0,850 & & & \\
\hline $\begin{array}{l}\text { Os setores da organização mantêm comunicação eficiente e rápida com outras } \\
\text { organizações. }\end{array}$ & 0,647 & & & \\
\hline $\begin{array}{l}\text { A organização tem mecanismos para avaliar, periodicamente, a adequação de sua } \\
\text { estratégia às demandas do ambiente externo. }\end{array}$ & & 0,798 & & \\
\hline $\begin{array}{l}\text { A organização tem mecanismos para levar em conta, sistematicamente, as } \\
\text { demandas do ambiente externo em suas decisões. }\end{array}$ & & 0,789 & & \\
\hline $\begin{array}{l}\text { A estratégia de atuação organizacional reflete bem as demandas do ambiente } \\
\text { externo. }\end{array}$ & & 0,747 & & \\
\hline
\end{tabular}




\section{Tabela 3 (continuação)}

\begin{tabular}{|c|c|c|c|c|}
\hline \multirow{2}{*}{ Itens* } & \multicolumn{4}{|c|}{ Fatores } \\
\hline & 1 & 2 & 3 & 4 \\
\hline $\begin{array}{l}\text { Alguns grupos influentes, externos à organização, acham que esta deve adotar uma } \\
\text { forma diferente de estruturar seu funcionamento. }\end{array}$ & & & 0,577 & \\
\hline $\begin{array}{l}\text { Alguns grupos influentes, internos, acham que a organização deve ter missão } \\
\text { completamente distinta da atual. }\end{array}$ & & & 0,571 & \\
\hline Alguns grupos da organização apresentam interesse em promover mudanças. & & & 0,563 & \\
\hline $\begin{array}{l}\text { A organização atua em um contexto externo em que a inovação tecnológica é } \\
\text { constante. }\end{array}$ & & & & 0,573 \\
\hline A organização é bastante sensível às interferências do governo. & & & & 0,572 \\
\hline $\begin{array}{l}\text { Novas regulamentações de governo (novas legislações, novas prioridades de } \\
\text { cumprimento de leis, desregulamentações) afetam diretamente a organização. }\end{array}$ & & & & 0,627 \\
\hline $\begin{array}{l}\text { Os choques na economia (quebra de mercado de valores, flutuações nas taxas de } \\
\text { juros, flutuações nas taxas de câmbio etc.) afetam diretamente essa organização. }\end{array}$ & & & & 0,609 \\
\hline Alfas de Cronbach & $\mathbf{0 , 9 6}$ & $\mathbf{0 , 7 5}$ & $\mathbf{0 , 7 8}$ & $\mathbf{0 , 7 5}$ \\
\hline
\end{tabular}

Nota. * Foram colocados apenas os itens mais ilustrativos.

A segunda escala apresenta os resultados dos fatores propulsores de mudanças transacionais (1) práticas gerenciais; (2) autonomia das unidades e pessoas; (3) o trabalho em grupo; e (4) burocracia e continuísmo - de acordo Neiva e Domingos (2011). Essa escala encontra-se apresentada na Tabela 4.

Tabela 4

\section{Estrutura Fatorial dos Fatores Transacionais que Facilitam ou Inibem a Mudança Organizacional}

\begin{tabular}{|c|c|c|c|c|}
\hline \multirow{2}{*}{ Itens* } & \multicolumn{4}{|c|}{ Fatores } \\
\hline & 1 & 2 & 3 & 4 \\
\hline Os gerentes direcionam as equipes de trabalho para o alcance de resultados. & 0,910 & & & \\
\hline Os gerentes expõem com clareza os objetivos a serem alcançados pela equipe. & 0,910 & & & \\
\hline $\begin{array}{l}\text { Os gerentes têm clareza da direção e das metas definidas pela organização para o } \\
\text { cumprimento das atividades sob sua responsabilidade. }\end{array}$ & 0,871 & & & \\
\hline Os gerentes têm uma visão clara sobre os objetivos da organização. & 0,867 & & & \\
\hline $\begin{array}{l}\text { Os gerentes estimulam as equipes a buscar conhecimentos necessários à realização } \\
\text { do trabalho. }\end{array}$ & 0,859 & & & \\
\hline $\begin{array}{l}\text { As pessoas têm liberdade para realizar o trabalho de forma diferente das normas } \\
\text { estabelecidas. }\end{array}$ & & 0,709 & & \\
\hline $\begin{array}{l}\text { A autonomia das unidades organizacionais (setores, departamentos) lhes permite } \\
\text { implantar mudanças de forma independente da alta direção da organização. }\end{array}$ & & 0,566 & & \\
\hline As pessoas têm liberdade para decidir como realizar as tarefas a elas atribuídas. & & 0,555 & & \\
\hline A quantidade de níveis hierárquicos da organização é pequena. & & 0,531 & & \\
\hline $\begin{array}{l}\text { Alguns projetos são realizados por meio da participação conjunta de várias } \\
\text { unidades / setores organizacionais. }\end{array}$ & & & 0,519 & \\
\hline O trabalho está organizado para ser realizado em equipe. & & & 0,517 & \\
\hline $\begin{array}{l}\text { Existem equipes destinadas a desenvolver projetos inovadores, hierarquicamente } \\
\text { vinculadas à alta direção da organização. }\end{array}$ & & & 0,538 & \\
\hline
\end{tabular}




\section{Tabela 4 (continuação)}

\begin{tabular}{|c|c|c|c|c|}
\hline \multirow{2}{*}{ Itens* } & \multicolumn{4}{|c|}{ Fatores } \\
\hline & 1 & 2 & 3 & 4 \\
\hline Na realização das tarefas, predomina o excesso de regras a serem cumpridas. & & & & $-0,783$ \\
\hline $\begin{array}{l}\text { Na realização das tarefas, predomina a repetição de ações desnecessárias pelos } \\
\text { servidores. }\end{array}$ & & & & $-0,672$ \\
\hline Há excesso de burocracia a cumprir na realização do trabalho. & & & & $-0,651$ \\
\hline Alfas de Cronbach & 0,95 & $\mathbf{0 , 7 9}$ & $\mathbf{0 , 7 6}$ & $\mathbf{0 , 7 5}$ \\
\hline
\end{tabular}

Nota. * Foram colocados apenas os itens mais ilustrativos.

\section{Instrumento de percepção de mudanças organizacionais}

Para exame da validade estatística do instrumento, foi realizada uma análise fatorial no intuito de verificar se a estrutura corresponde aos dois tipos de mudança organizacional avaliados. O primeiro passo para a análise fatorial foi avaliar a adequação e a fatorabilidade da amostra. A matriz apresentou mais de 50\% das correlações significativas acima de 0.40; KMO - (Kaiser-Meyer-Olkin Measure of Sampling Adequacy) - de 0,93 e determinante bem próximo de zero.

De acordo com os resultados, a variância total explicada pelo instrumento composto de trinta itens agregados em dois fatores, foi de $48 \%$. Os fatores extraídos foram denominados de Mudança Transformacional e Mudança Transacional, e suas confiabilidades internas avaliadas pelo cálculo da média aritmética da correlação item-total e pelo Alfa de Cronbach.

Os dezoito itens relacionados à percepção de Mudança Transformacional apresentaram cargas fatoriais variando entre 0,513 e 0,856, correlação item-fator com média de 0,67 e Alfa de Cronbach de 0,93 . Os itens relacionados à percepção de Mudança Transacional apresentaram cargas fatoriais variando entre 0,584 e 0,718, correlação item-fator, com média de 0,41 e Alfa de Cronbach 0,76. A Tabela 5 apresenta a análise fatorial do instrumento de percepção de mudanças - Fator Mudança Transformacional, os itens que o compõem, suas respectivas cargas fatoriais e o Alfa de Cronbach. A Tabela 6 apresenta a análise fatorial do instrumento de percepção de mudanças - Fator Mudança Transacional, os itens que o compõem, suas respectivas cargas fatoriais e o Alfa de Cronbach.

Tabela 5

\section{Análise Fatorial do Instrumento de Percepção de Mudanças - Fator Mudança Transformacional}

\begin{tabular}{clc}
\hline \multicolumn{1}{c}{ Fator Mudança Transformacional - Alfa de Cronbach = 0,93 } \\
\hline Item & Descrição & Carga fatorial \\
\hline 01 & As mudanças que ocorreram afetaram toda a Instituição. & 0,577 \\
05 & As mudanças eram necessárias para a sobrevivência da Instituição. & 0,589 \\
12 & As mudanças ocorreram porque era preciso mudar. & 0,677 \\
14 & As mudanças afetaram a Instituição de cima abaixo. & 0,646 \\
15 & Hoje as pessoas dizem que a Instituição é outra. & 0,762 \\
16 & Agora os valores são completamente diferentes do que eram. & 0,790 \\
17 & Efetuar a mudança era uma intenção da Instituição. & 0,593 \\
18 & Houve uma mudança radical em como as coisas eram feitas na Instituição. & 0,789 \\
19 & Com as mudanças, as pessoas mudaram seus comportamentos. & 0,689 \\
\hline
\end{tabular}


Tabela 5 (continuação)

\begin{tabular}{|c|c|c|}
\hline \multicolumn{3}{|c|}{ Fator Mudança Transformacional - Alfa de Cronbach $=\mathbf{0 , 9 3}$} \\
\hline Item & Descrição & Carga fatorial \\
\hline 20 & As mudanças aconteceram ao mesmo tempo em todos os setores da Instituição. & 0,687 \\
\hline 21 & As orientações tradicionais da Instituição foram abandonadas. & 0,513 \\
\hline 22 & Hoje a cultura da Instituição é outra. & 0,699 \\
\hline 24 & As mudanças foram muito abrangentes. & 0,856 \\
\hline 25 & Com as mudanças, a Instituição tomou outro direcionamento. & 0,806 \\
\hline 27 & Houve a implantação de novas tecnologias. & 0,635 \\
\hline 28 & Com as mudanças, houve melhora no atendimento ao cliente. & 0,750 \\
\hline 29 & O desempenho dos empregados no trabalho melhorou muito com as mudanças. & 0,738 \\
\hline 30 & As mudanças alteraram a visão da Instituição. & 0,833 \\
\hline
\end{tabular}

Tabela 6

Análise Fatorial do Instrumento de Percepção de Mudanças - Fator Mudança Transacional

\begin{tabular}{clc}
\hline \multicolumn{2}{c}{ Fator Mudança Transacional - Alfa de Cronbach = 0,76 } & \\
\hline Item & Descrição & Carga fatorial \\
\hline 02 & As mudanças afetaram apenas algumas áreas. & 0,583 \\
03 & As mudanças alteraram somente os procedimentos burocráticos. & 0,504 \\
04 & As mudanças alteraram somente a forma de se trabalhar. & 0,461 \\
06 & As mudanças foram acontecendo gradualmente. & 0,622 \\
07 & Vira e mexe ocorrem pequenos ajustes no funcionamento da Instituição. & 0,545 \\
08 & As mudanças foram superficiais. & 0,718 \\
10 & Ocorreram pequenas alterações na estrutura da Instituição. & 0,584 \\
11 & As mudanças foram apenas de fachada. & 0,587 \\
13 & As alterações ocorreram, mas tudo voltou a ser o que era antes. & 0,582 \\
23 & Nessa Instituição, as mudanças estão sempre acontecendo. & 0,706 \\
26 & Ocorreram mudanças somente no nível gerencial. & 0,603 \\
\hline
\end{tabular}

\section{Dados descritivos da organização estudada}

Para análise do comportamento das variáveis, foram calculadas as medidas descritivas em função de $\mathrm{N}=340$. A variável Turbulência no Ambiente Externo apresentou valor de média um pouco mais elevado $(\mathrm{M}=2,13)$, superior ao ponto médio 2 . A escala variou de 0 a 4 pontos. A Tabela 7 apresenta o comportamento médio das variáveis de capacidade organizacional para mudanças. 
Tabela 7

Comportamento Médio das Variáveis de Capacidade Organizacional para Mudança

\begin{tabular}{lcc}
\hline & Capacidade para Mudança Transformacional & \\
\hline Características & Média & Desvio Padrão \\
\hline Estratégia de Atuação & 1,70 & 0,74 \\
Fluxo de Comunicação & 1,71 & 0,69 \\
Dinâmica de Coalizões & 1,87 & 0,47 \\
Turbulência no Ambiente Externo & 2,13 & 0,59 \\
Autonomia de Unidades e Pessoas & 1,55 & 0,66 \\
Trabalho em Grupo & 1,82 & 0,74 \\
Práticas Gerenciais & 2,00 & 0,75 \\
Burocracia e Continuísmo & 2,40 & 0,57 \\
\hline
\end{tabular}

Nota. $N=340$.

A variável Burocracia e Continuísmo apresentou valor de média mais elevado $(M=2,40)$, superior ao ponto médio 2. Quanto à percepção de mudança, a média aritmética do fator Mudança Transformacional foi de 4,31 e desvio padrão de $(\mathrm{SD}=2,16)$, sendo que a escala variou de 0 a 10 . Em relação à percepção de Mudança Transacional, a média aritmética apresentada foi de 5,11 e desvio padrão de $(\mathrm{SD}=1,69)$.

Os dados mostram que os respondentes perceberam em baixo grau, mudanças no órgão pesquisado. As Mudanças Transacionais foram percebidas de uma maneira mais acentuada que as Mudanças Transformacionais.

\section{Resultados do teste das relações entre as variáveis}

Inicialmente foi testada a relação entre todos os fatores de capacidade organizacional para mudanças e todas as mudanças percebidas. Quando analisados conjuntamente, somente os fatores Estratégia de Atuação e Dinâmica de Coalizões se apresentam como preditores dos dois tipos de mudanças organizacionais, apresentando $\mathrm{R}^{2}$ de $0,34(\mathrm{p}<0,002)$ para mudanças transacionais e $\mathrm{R}^{2}$ de $0,60(\mathrm{p}=0,000)$ para mudanças transformacionais.

Posteriormente, foi testado o modelo vinculado aos fatores de capacidade organizacional para mudanças transformacionais. Tal procedimento foi realizado com objetivo de investigar o poder preditivo dos fatores transformacionais na percepção de mudanças transformacionais.

Os resultados obtidos indicam que, de forma conjunta, os fatores transformacionais Estratégia de Atuação, Dinâmica de Coalizões, Fluxo de Comunicação explicam 35\% da variância da variável critério. $\mathrm{O}$ fator Turbulência no Ambiente Externo não apareceu como preditor. Com intuito de avaliar o papel de cada preditor, analisou-se o valor do Beta $(\beta)$. Os efeitos isolados desses fatores são apresentados na Tabela 8.

No teste do modelo, vinculado aos fatores de capacidade organizacional para mudanças transacionais, os resultados demonstraram o fator Trabalho em Grupo como preditor, apresentando com $\mathrm{R}^{2}$ de 0,163 e $\beta=0,251, \mathrm{p} \leq 0,000$. Os demais fatores não apareceram como preditores. Todas as correlações foram positivas entre as variáveis preditoras e as variáveis-critério. Os resultados dessa análise são apresentados por meio da Tabela 8. 
Tabela 8

\section{Resultados da Análise de Regressão Linear para Variáveis Preditoras de Percepção de Mudança Transformacional e Transacional}

\begin{tabular}{cccc}
\hline \multicolumn{4}{c}{ Percepção de Mudança Transformacional (VD) } \\
\hline Variável Preditora & $\mathbf{R}^{\mathbf{2}}$ & Beta $(\boldsymbol{\beta})$ & Sig \\
Estratégia de Atuação & 0,322 & 0,398 & 0,000 \\
Dinâmica de Coalizões & 0,341 & 0,129 & 0,010 \\
Fluxo de Comunicação & 0,354 & 0,161 & 0,011 \\
\hline & Percepção de Mudança Transacional (VD) & \\
\hline Variável Preditora & $\mathbf{R}^{\mathbf{2}}$ & Beta $(\boldsymbol{\beta})$ & Sig \\
Trabalho em Grupo & 0,163 & 0,251 & 0,000 \\
\hline
\end{tabular}

De acordo com os resultados apresentados anteriormente, percebe-se que, dos quatro fatores de capacidade organizacional para mudanças transformacionais, a Estratégia de Atuação, a Dinâmica de Coalizões e o Fluxo de Comunicação são percebidos pelos respondentes como relacionados positivamente à existência de mudanças transformacionais na organização.

No que diz respeito às mudanças transacionais, os resultados mostram que, dos quatro fatores de capacidade organizacional para mudanças transacionais, apenas o Trabalho em Grupo é percebido pelos respondentes como relacionado positivamente à existência de mudanças transacionais no órgão analisado. As variáveis Práticas Gerenciais, Autonomia de Unidades e Pessoas, Burocracia e Continuísmo não são percebidas como vinculadas à existência de mudanças transacionais.

Quando todos os fatores de capacidade organizacional para mudanças são colocados na regressão, os fatores hipoteticamente associados às mudanças transacionais não apresentam efeito sobre a percepção de mudanças, cedendo espaço para os fatores associados às mudanças transformacionais.

\section{Discussão e Conclusões}

Pela análise dos dados coletados, os respondentes não percebem a presença dos fatores facilitadores de mudanças organizacionais. Além de não perceberem a existência desses fatores, os respondentes também não percebem a existência de mudanças, o que difere dos relatos obtidos com os informantes-chave. Esse resultado está em consonância com os resultados encontrados por Neiva e Paz (2007), que identificou que os órgãos públicos apresentam índices baixos nos fatores de capacidade organizacional para mudanças.

Tal qual a maioria das instituições públicas, o órgão analisado é gerido sob estritas regras de trabalho, em que a prevalência do sistema burocrático e de formas de atuação vinculadas a procedimentos de controle são preponderantes. Essa característica é entendida como forte entrave à ocorrência de processos de mudança transacional (Burke, 2011a; Burke \& Litwin, 1992; Neiva \& Paz, 2007) e tende a ser percebida pelo indivíduo como a manutenção de formas de trabalho que impedem a evolução individual e a do órgão como um todo.

A existência da excessiva burocracia e de regras nas formas de trabalho, a formalização e a padronização de procedimentos, a repetição de práticas rotineiras e a impossibilidade das pessoas tomarem decisões importantes relativas aos seus trabalhos, levam o respondente a perceber que nada 
foi alterado ao longo do tempo e, portanto, não há a identificação da ocorrência de mudanças transacionais naquele ambiente organizacional.

Contudo, o objetivo principal do trabalho foi testar as relações entre capacidade organizacional para mudanças e as mudanças percebidas pelos membros da organização. A influência positiva da Estratégia de Atuação na ocorrência de mudança corrobora os achados de Neiva e Domingos (2011); Judge e Douglas (2009); Gravenhorst et al. (2003); Huber, Sutcliffe, Miller e Glick (1993). Também exercem influência a Dinâmica de Coalizões, corroborando os trabalhos de Greenwood e Hinings (1996); de Bouckenooghe et al. (2009) e de Choi e Ruona (2011). O Fluxo de Comunicação também apareceu como preditor, indo ao encontro dos achados de Judge e Douglas (2009); Walker, Armenakis e Bernerth (2007); Soparnot (2011); Gravenhorst et al. (2003), Bressan (2001); Neiva e Paz (2007); Damanpour (1991); Mignerey, Rubin e Gorden (1995).

Para as mudanças Transformacionais, além dos fatores Estratégia de Atuação e Dinâmica de Coalizões, o Fluxo de Comunicação igualmente permanece como preditor. Tais resultados corroboram os trabalhos de Walker et al. (2007) e Bressan (2001) que enfatizam a comunicação aberta e honesta entre agentes de mudança e membros de toda a organização.

Em relação à percepção de mudança do tipo Transacional, o Trabalho em Grupo aparece como o único preditor, sendo que as Práticas Gerenciais, a Autonomia de Unidades e Pessoas, a Burocracia e Continuísmo não são preditores desse tipo de mudança.

Na amostra analisada, a variável Turbulência no Ambiente Externo não é percebida como relacionada à existência de mudanças transformacionais, não confirmando assim os achados de Huber et al. (1993) e de Mintzberg e Westley (1992).

Os autores acreditam que os fatores de capacidade organizacional para mudanças transformacionais possuem influência considerável sobre as mudanças percebidas na amostra avaliada. A ausência da influência do fator Turbulência no Ambiente Externo sobre as mudanças percebidas pode estar relacionada ao fato da organização estudada estar inserida em um ambiente externo de relativa complexidade, mas com pouco dinamismo, e de se tratar de um órgão público de execução.

A afirmação de Gravenhorst et al. (2003) de que o grau em que aspectos da organização e aspectos do processo vão contribuir ou restringir mudanças está diretamente relacionado com a capacidade de mudança da organização, ficam aqui constatados.

De acordo com Greenwood e Hinings (1996), existe relacionamento entre as mudanças e a maneira como a organização mobiliza os recursos internos para que elas ocorram. Assim, é possível perceber que esses autores estavam certos quando afirmaram ser a mudança algo difícil de ocorrer devido à dificuldade da organização mobilizar suporte ou recursos internos que estejam positivamente associados à introdução de mudanças organizacionais.

Dessa forma, segundo o modelo, para a ocorrência de mudanças transacionais, é necessário que o órgão analisado minimize os procedimentos burocráticos e o continuísmo, reduza os níveis hierárquicos existentes, e facilite e incentive a adoção de inovações nos processos de trabalho.

Este trabalho colabora para ampliação do conhecimento na área e enfatiza a necessidade dos gestores responsáveis por processos de mudanças organizacionais em órgão públicos e privados, em avaliar a existência dos fatores de capacidade organizacional para mudanças e a sua influência na consecução desse processo. Do ponto de vista científico, contribui para sistematizar o construto de capacidade organizacional para mudanças e especifica fatores que seriam consequentes do fenômeno. Avança em aspectos importantes na área da mudança organizacional, por se caracterizar em uma abordagem quantitativa e sistematizada.

Apesar da amostra se concentrar em uma única organização, a pesquisa apresenta uma amostra representativa em termos numéricos e de variabilidade (indicada por suas características), dando 
sustentação empírica para os trabalhos de Gravenhorst et al. (2003), Judge e Elenkov (2005), Judge e Douglas (2009) e Soparnot (2011).

\section{Referências}

Armenakis, A. A., \& Bedeian, A. G. (1999). Organizational change: a review of theory and research in the 1990s. Journal of Management, 25(3), 293-315. doi: 10.1177/014920639902500303

Barnett, W. P., \& Carroll, G. R. (1995). Modeling internal organizational change. Annual Review of Sociology, 21, 217-236. doi: 10.1146/annurev.so.21.080195.001245

Bouckenooghe, D., Devos, G., \& Van Den Broeck, H. (2009). Organizational change questionnaire climate of change, processes, and readiness: development of a new instrument. The Journal of Psychology, 143(6), 559-599. doi: 10.1080/00223980903218216

Bressan, C. L. (2001). Uma contribuição à compreensão do fenômeno de mudança organizacional a partir da percepção gerencial (Dissertação de mestrado). Universidade de Brasília, Brasília, DF, Brasil.

Buono, A. F., \& Kerber, K. W. (2010). Creating a sustainable approach to change: building organizational change capacity. SAM Advanced Management Journal, 75(2), 4-22.

Burke, W. W. (2011b). A perspective on the field of organization development and change: the zeigarnik effect. Journal of Applied Behavioral Science, 47(2), 143-167. doi: $10.1177 / 0021886310388161$

Burke, W. W. (2011a). Organization change: theory and practice (3rd ed.). Los Angeles: Thousand Oaks, Sage Publications.

Burke, W. W., \& Litwin, G. (1992). A causal model of organizational performance and change. Journal of Management, 18(3), 523-545. doi: 10.1177/014920639201800306

Choi, M., \& Ruona, W. E. A. (2011). Individual readiness for organizational change and its implications for human resource and organization development. Human Resource Development Review, 10(1), 46-73. doi: 10.1177/1534484310384957

Cunningham, C. E, Woodward, C. A., Shannon, H. S., Macintosh, J., Lendrum, B., Rosenbloom, D., \& Brown, J. (2002). Readiness for organizational change: a longitudinal study of workplace, psychological, and behavioral correlates. Journal of Occupational and Organizational Psychology, 75(4), 377-392. doi: 10.1348/096317902321119637

Damanpour, F. (1991). Organizational innovation: a meta-analysis of effect of determinants and moderators. Academy of Management Journal, 34(3), 555-590. doi: 10.2307/256406

Danna, E. C. (2012). Capacidade organizacional para mudança: estudo de caso de uma organização pública legislativa (Dissertação de mestrado). Universidade de Brasília, Brasília, DF, Brasil.

Eisenhardt, K. M., \& Martin, J. A. (2000). Dynamic capabilities: what are they? Strategic Management Journal, 21(10/11), 1105-1122. doi: 10.1002/10970266(200010/11)21:10/11<1105::AID-SMJ133>3.0.CO;2-E

Fischer, H. C. R., \& Lima, S. M. V. (2005). Validação de instrumento para diagnóstico de condições facilitadoras de mudança organizacional. Revista Psicologia Organizações e Trabalho, 5(1), 1344. 
Glor, E. D. (2007). Assessing organizational capacity to adapt. Emergence: Complexity and Organization, 9(3), 33-46.

Gravenhorst, K. M. B, Werkman, R. A., \& Boonstra, J. J. (2003). The change capacity of organisations: general assessment and five configurations. Applied Psychology: An International Review, 52(1), 83-105. doi: 10.1111/1464-0597.00125

Greenwood, R., \& Hinings, C. R. (1996). Understanding radical organizational change: bringing together the old and the new institutionalism. Academy of Management Review, 21(4), 10221054. doi: 10.5465/AMR.1996.9704071862

Hatum, A., \& Pettigrew, A. M. (2004). Adaptive responses under competitive pressure: organizational flexibility in an emergent economy. Management Research: The Journal of the Iberoamerican Academy of Management, 2(2), 97-114. doi: 10.1108/15365430480000504

Huber, G. P., Sutcliffe, K., Miller, C. C., \& Glick, W. H. (1993). Understanding and predicting organizational change. In G. P. Huber \& W. H. Glick (Eds.), Organizational change and redesign (pp. 214-454). New York: Oxford University Press.

Judge, W. Q., \& Blocker, C. P. (2008). Organizational capacity for change and strategic ambidexterity: flying the plane while rewiring it. European Journal of Marketing, 42(9/10), 915-926. doi: 10.1108/03090560810891073

Judge, W. Q., \& Douglas, T. (2009). Organizational change capacity: the systematic development of scale. Journal of Organizational Change Management, 22(6), 635-649. doi: $10.1108 / 09534810910997041$

Judge, W. Q., \& Elenkov, D. (2005). Organizational capacity for change and environmental performance: an empirical assessment of Bulgarian firms. Journal of Business Research, 58(7), 893-901. doi: 10.1016/j.jbusres.2004.01.009

Lima, S. M. V., \& Bressan, C. L. (2003). Mudança organizacional: uma introdução. In S. M. V. Lima (Org.), Mudança organizacional; teoria e gestão (pp. 17-63). Rio de Janeiro: FGV.

Litaker, D., Ruhe, M., \& Flocke, S. (2008). Making sense of primary care practices' capacity for change. Translational Research, 152(5), 245-253. doi: 10.1016/j.trsl.2008.09.005

Mignerey, J. T., Rubin, R. B., \& Gorden, W. I. (1995). Organizational entry: an investigation of newcomer communication behavior and uncertainty. Comunication Research, 22(1), 54-86. doi: $10.1177 / 009365095022001003$

Mintzberg, H., \& Westley, F. (1992). Cycles of organizational change. Strategic Management Journal, 13(2), 39-59. doi: 10.1002/smj.4250130905

Neiva, E. R., \& Domingos, S. G. (2011, setembro). Validação de instrumento para avaliação da capacidade organizacional para a mudança. Anais do Encontro Nacional da Associação Nacional de Pós-Graduação e Pesquisa em Administração, Rio de Janeiro, RJ, Brasil, 35.

Neiva, E. R., \& Paz, M. G. T. da (2007). Percepção de mudança organizacional: um estudo em uma organização pública brasileira. Revista de Administração Contemporânea, 11(1), 31-52. doi: $10.1590 / \mathrm{S} 1415-65552007000100003$

Neiva, E. R., \& Paz, M. G. T. da (2012). Percepção de mudança individual e organizacional: o papel das atitudes, dos valores, do poder e da capacidade organizacional. Revista de Administração, 47(1), 22-37. doi: 10.5700/rausp1023

Oxtoby, B., Mcguiness, T., \& Morgan, R. (2002). Developing organizational change capability. European Management Journal, 20(3), 310-320. doi: 10.1016/S0263-2373(02)00047-6 
Pettigrew, A. M., Woodman, R. W., \& Cameron, K. S. (2001). Studying organizational change and development: challenges for future research. Academy of Management Journal, 44(4), 697-713. doi: $10.2307 / 3069411$

Prochaska, J. O., Norcross, J. C., \& DiClemente C. C. (1994). Changing for good: the revolutionary program that explains the six stages of change and teaches you how to free yourself from bad habits. New York: W. Morrow.

Sampieri, R. H., Collado, C. F., \& Lúcio, P. B. (2006). Metodologia de pesquisa (3a ed.). São Paulo: McGraw Hill.

Slack, A. J., \& Hinings, C. R. (2004). Strategic change and the role of interests, power and organizational capacity. Journal of Sport Management, 18(2), 158-198.

Soparnot, R. (2011). The concept of organizational change capacity. Journal of Organizational Change Management, 24(5), 640-661. doi: 10.1108/09534811111158903

Staber, U., \& Sydow, J. (2002) Organizational adaptive capacity; a structuration perspective. Journal of Management Inquiry, 11(4), 408-424. doi: 10.1177/1056492602238848

Van de Ven, A., \& Poole, M. S. (2005). Alternative approaches for studying organizational change. Organization Studies, 26(9), 1377-1404. doi: 10.1177/0170840605056907

Walker, H. J., Armenakis, A. A., \& Bernerth, J. B. (2007). Factors influencing organizational change efforts: an integrative investigation of change content, context, process and individual differences. Journal of Organizational Change Management, 20(6), 761-773. doi: 10.1108/09534810710831000

Weick, K. E., \& Quinn, R. E. (1999). Organizational change and development. Annual Review of Psychology, 50, 361-386. doi: 10.1146/annurev.psych.50.1.361

Weiner, B. J., Amick, H., \& Lee, S. Y. D. (2008). Conceptualization and measurement of organizational readiness for change: a review of the literature in health services research and other fields. Medical Care Research and Review, 65(4), 379- 436. doi: $10.1177 / 1077558708317802$

Woodman, R. W. (1989). Evaluation research in organizational change: arguments for a "combined paradigm" approach. In R. W. Woodman \& W. A. Pas-More (Eds.), Research in Organizational Change and Development (Vol. 3, pp. 161-180). Greenwich, CT: JAI Press. 